D.T. Wickramasinghe, L. Ferrario, and G.V. Bicknell, eds.

\title{
Magnetohydrodynamic Turbulence in Accretion Discs: Towards More Realistic Models
}

\author{
Ulf Torkelsson \\ Institute of Astronomy, Madingley Road, Cambridge CB3 9JN, United \\ Kingdom
}

\author{
Axel Brandenburg \\ Department of Mathematics, University of Newcastle upon Tyne, NE1 \\ $7 R U$, United Kingdom
}

\author{
$\AA$ ke Nordlund \\ Theoretical Astrophysics Center, Juliane Maries vej 30, DK-2100 \\ Copenhagen $\varnothing$, Denmark \\ Robert F. Stein \\ Department of Physics and Astronomy, Michigan State University, East \\ Lansing, MI 48824, USA
}

\begin{abstract}
The shearing box has rapidly become the accepted way to investigate turbulence in Keplerian shear flows. In this paper we discuss to what extent and in which way the outcome of the shearing box is affected by the adopted boundary conditions, and how the shearing box can be modified to capture more of the physics of an accretion disc. The original shearing box model is too symmetric to generate a net accretion flow, but the symmetry can be broken by including the main effects of the cylindrical geometry of the real disc. However the quantitative change in the resulting angular momentum transport is small.
\end{abstract}

Keywords: accretion: accretion discs - MHD - turbulence - magnetic fields

\section{Introduction}

Balbus \& Hawley (1991) suggested that a magnetic shearing instability is the cause of turbulence in accretion discs. This suggestion appeared attractive as, in spite of several attempts, it had not been possible to find a hydrodynamic instability that could give rise to turbulence in a Keplerian accretion disc (e. g. Balbus this volume). To estimate the efficiency of the shear-induced turbulence as a transporter of angular momentum it is necessary to resort to numerical simulations. A small part of the accretion disc is approximated by a Cartesian box with a linear shear flow (Hawley, Gammie \& Balbus 1995, 1996, Matsumoto \& Tajima 1995, Brandenburg, Nordlund, Stein \& Torkelsson 1995 hereafter BNST, Stone, Hawley, Gammie \& Balbus 1996 hereafter SHGB; see also Hawley this volume). Simulations have been done both with and without taking into ac- 
count the vertical stratification of the box, and with different vertical boundary conditions.

In the rest of this paper we will discuss the importance of differences in the vertical boundary conditions (Sect. 2) and how the symmetry of the shearing box can be broken by including the major effects of the more natural cylindrical geometry (Sect. 3). Sect. 4 contains a brief discussion and our main conclusions.

\section{The influence of the boundary conditions}

The ordinary approach to simulating turbulence in accretion discs is to approximate a small part of the accretion disc with a shearing box. The shearing box is a Cartesian box rotating with the angular velocity $\Omega_{0}$ characteristic of the Keplerian rotation at a distance $R_{0}$ from the central object. $x, y$ and $z$ are the radial, azimuthal and vertical coordinates in the frame of the box. The shear is linearised around the radius $R_{0}$, such that $\mathbf{u}_{\text {shear }}=-3 / 2 x \Omega_{0} \hat{\mathbf{y}}$, and the magnetohydrodynamic equations are solved for the density, internal energy, magnetic field, and the deviation from the shear flow. The boundary conditions of the box are chosen as periodic in $y$, and sliding periodic in $x$, that is $F\left(\frac{1}{2} L_{x}, y, z\right)=F\left(-\frac{1}{2} L_{x}, y+\frac{3}{2} \Omega_{0} L_{x} t, z\right)$, where $L_{x}$ is the radial extent of the box and $F$ is any quantity that we are solving for in the simulation. The vertical boundary conditions can be chosen in various ways, e.g. periodic boundary conditions (SHGB), or stress-free boundaries with $\mathbf{u}_{z}=0$ and a purely vertical magnetic field (BNST). The boundary conditions have little influence locally, but determine the global properties of the magnetic field. A toroidal magnetic flux can be produced by the sliding periodic radial boundary conditions, which shear out a radial magnetic flux. The radial magnetic flux is conserved if both the azimuthal and vertical boundaries are (sliding) periodic, as in SHGB, but not with the boundary conditions of BNST. The vertical magnetic flux is conserved in all simulations as a result of the (sliding) periodic boundary conditions on the azimuthal (radial) boundaries.

As neither the azimuthal nor the radial magnetic flux is conserved in our simulations, it is possible for us to look for a large-scale dynamo. An oscillatory dynamo, whose azimuthal magnetic field reverses direction every $\sim 30$ orbital periods, appears in our stratified shearing box (BNST Fig. 2). The oscillations are however fragile in the sense that they do not appear in an unstratified box, although a net azimuthal flux is still generated (Fig. 1).

\section{The tension of the toroidal magnetic field}

The major limitation of the shearing box is that it is more symmetric than a real accretion disc. Consequently there is no real accretion flow in a shearing box, although there is a stress, from which the angular 'momentum transport in an accretion disc can be calculated. Four additional force terms appear in cylindrical coordinates:

- The centrifugal acceleration, $u_{\phi}^{2} / r$

- The tension of the toroidal magnetic field, $-B_{\phi}^{2} /\left(\mu_{0} \rho r\right)$ 


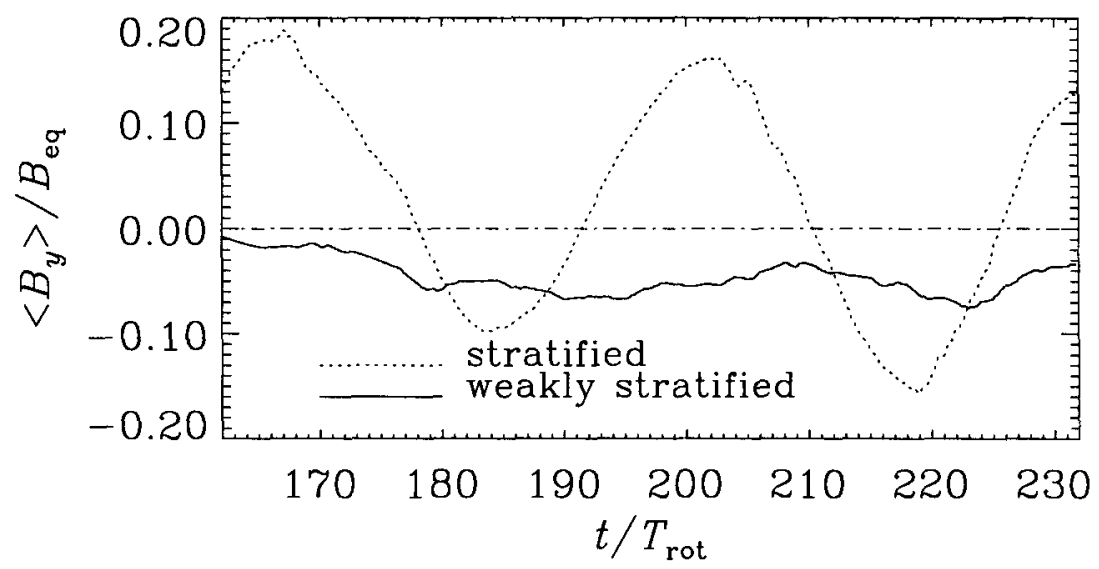

Figure 1. The time evolution of the mean toroidal magnetic field in an unstratified shearing box (solid line) and a stratified shearing box (dashed line). Magnetic field is given in units of the field strength required for equipartition between magnetic and thermal energy, and time is given in orbital periods
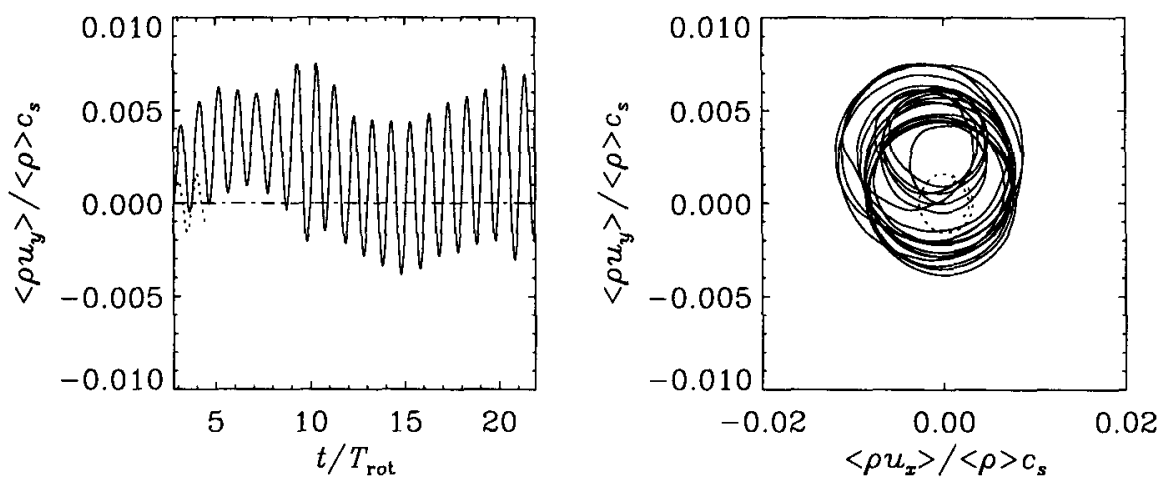

Figure 2. $\left\langle\rho u_{y}\right\rangle$ versus time (left), and $\left\langle\rho u_{y}\right\rangle$ versus $\left\langle\rho u_{x}\right\rangle$ (right) normalised by $\langle\rho\rangle c_{\mathrm{s}}$. The dashed line is for a standard shearing box, whereas the solid line represents a model taking into account the effects discussed in Sect. 3 
- The azimuthal acceleration, $-u_{r} u_{\phi} / r$

- The azimuthal tension of the magnetic field, $B_{r} B_{\phi} /\left(\mu_{0} \rho r\right)$

In particular the tension of the toroidal magnetic field can become strong, and will always provide a force directed inwards, thus breaking the symmetry of the shearing box and introducing a real accretion flow. Averaging the momentum density over the box an epicyclic motion appears already in the ordinary shearing box simulations (cf. BNST Fig. 2), although it is much smaller than the random turbulent motions. The size of the epicyclic motion seen in Fig. 1a of Brandenburg et al. (1996) was about ten times too big due to an error in the code, but the average accretion flow and other relevant quantities were not affected by this error. A corrected version of the code shows that the symmetry breaking terms lead to only a small increase in the amplitude of the motion, but the center of the motion is displaced to $\left(\left\langle\rho u_{x}\right\rangle,\left\langle\rho u_{y}\right\rangle\right) /\langle\rho\rangle c_{\mathrm{s}} \approx(-0.001,0.003)$ (Fig. 2), because the Coriolis force needs to balance the newly introduced magnetic forces.

\section{Discussion}

The numerical simulations of MHD-turbulence in accretion discs have now reached the stage where they agree on the overall properties of the turbulence (cf. SHGB and BNST). In particular the local properties of the turbulence appear to be largely insensitive to both the boundary conditions and modifications of the equations of the shearing box model. The currently favoured value for $\alpha_{\mathrm{SS}} \sim 0.01$ may however be too small to explain for instance dwarf nova outbursts. It is therefore worth noting that some recent work (Matsuzaki \& Matsumoto this volume) suggests that there exists a second regime for accretion discs. These discs are magnetically dominated, $\beta \sim 0.1$ and yield $\alpha_{\mathrm{SS}} \sim 0.1$.

Acknowledgments. UT is supported by an EU post-doctoral fellowship. This work was supported in part by the Danish National Research Foundation through its establishment of the Theoretical Astrophysics Center.

\section{References}

Balbus, S. A., Hawley, J. F. 1991, ApJ, 376, 214

Brandenburg, A., Nordlund, Å. Stein, R. F., Torkelsson, U. 1995, ApJ, 446, 741 (BNST)

Brandenburg, A., Nordlund, Å. Stein, R. F., Torkelsson, U. 1996, ApJ, 458, L45

Hawley, J. F., Gammie, C. F., Balbus, S. A. 1995, ApJ, 440, 742

Hawley, J. F., Gammie, C. F., Balbus, S. A. 1996, ApJ, 464, 690

Matsumoto, R., Tajima, T. 1995, ApJ, 445, 767

Stone, J. M., Hawley, J. F., Gammie, C. F., Balbus, S. A. 1996, ApJ, 463, 656 (SHGB) 


\section{Discussion}

S. Balbus: Could you clarify your boundary conditions on the velocity?

$U$. Torkelsson: The vertical velocity, $v_{z}$, and the derivatives of the horizontal velocity $\partial v_{x} / \partial z$ and $\partial v_{y} / \partial z$, are 0 .

C. Curry: Could you comment on the fact that both your group and Stone, Balbus et al. found that increasing grid resolution leads to larger values of $\alpha$ ?

U. Torkelsson: Our first simulations with a standard shearing box (ApJ, 446, 741) seem to have converged to values independent of the grid resolution. The later simulations (ApJ, 458, L45) give results that depend on the resolution.

$K$. Horne: Can you say anything yet about the vertical $(z)$ distribution of the heating (dissipation) per unit mass?

U. Torkelsson: The magnetic force seems to be largely independent of $z$, that is one can expect a more efficient heating in the upper layers.

C. Tout: Do you also agree that you can get large values for $\alpha$ for certain initial conditions? So that it is an imposed vertical field rather than just an initial condition?

U. Torkelsson: Yes, an imposed vertical magnetic flux gives high values for $\alpha_{\mathrm{SS}}$. The imposed vertical magnetic flux is conserved by the boundary conditions, so such a magnetic field is not only an initial condition. 